\title{
Viruses, SUMO, and immunity: the interplay between viruses and the host SUMOylation system
}

\author{
Fergan Imbert ${ }^{1} \cdot$ Dianne Langford $^{1} \mathbb{D}$
}

Received: 1 March 2021 / Revised: 19 June 2021 / Accepted: 29 June 2021 / Published online: 3 August 2021

(c) Journal of NeuroVirology, Inc. 2021

\begin{abstract}
The conjugation of small ubiquitin-like modifier (SUMO) proteins to substrates is a well-described post-translational modification that regulates protein activity, subcellular localization, and protein-protein interactions for a variety of downstream cellular activities. Several studies describe SUMOylation as an essential post-translational modification for successful viral infection across a broad range of viruses, including RNA and DNA viruses, both enveloped and un-enveloped. These viruses include but are not limited to herpes viruses, human immunodeficiency virus-1, and coronaviruses. In addition to the SUMOylation of viral proteins during infection, evidence shows that viruses manipulate the SUMO pathway for host protein SUMOylation. SUMOylation of host and viral proteins greatly impacts host innate immunity through viral manipulation of the host SUMOylation machinery to promote viral replication and pathogenesis. Other post-translational modifications like phosphorylation can also modulate SUMO function. For example, phosphorylation of COUP-TF interacting protein 2 (CTIP2) leads to its SUMOylation and subsequent proteasomal degradation. The SUMOylation of CTIP2 and subsequent degradation prevents CTIP2-mediated recruitment of a multi-enzymatic complex to the HIV-1 promoter that usually prevents the transcription of integrated viral DNA. Thus, the "SUMO switch" could have implications for CTIP2-mediated transcriptional repression of HIV-1 in latency and viral persistence. In this review, we describe the consequences of SUMO in innate immunity and then focus on the various ways that viral pathogens have evolved to hijack the conserved SUMO machinery. Increased understanding of the many roles of SUMOylation in viral infections can lead to novel insight into the regulation of viral pathogenesis with the potential to uncover new targets for antiviral therapies.
\end{abstract}

Keywords SUMOylation · Virus · Post-translational modifications · Infection

\section{Introduction}

The conjugation of the small ubiquitin-like modifier (SUMO) to proteins increases the diversity and complexity of the proteome in eukaryotic cells. The Saccharomyces cerevisiae SUMO homolog Smt 3 is the earliest identification of SUMO and was characterized as a suppressor of the centromeric protein Mif2. Still, the exact mechanism of suppression remained unclear even after its discovery (Meluh and Koshland 1995). The field grew in 1996 when several laboratories independently identified SUMO in mammals. One group identified SUMO as an interacting partner of DNA repair proteins RAD51/RAD52 in the nucleus (Shen et al. 1996), providing

Dianne Langford

tdl@temple.edu

1 Department of Neuroscience, Lewis Katz School of Medicine, Temple University, PA 19140 Philadelphia, USA more evidence regarding the potential role of SUMO in cellular functions. In addition, another group identified SUMO as a novel binding partner of the pro-apoptotic protein, Fas, during their studies of extrinsic apoptotic pathways (Okura et al. 1996). SUMO was also identified as the promyelocytic leukemia protein (PML) associated tumor-suppressor protein, regulating its localization and function (Boddy et al. 1996; Zhong et al. 2000). Together, these studies provided early evidence of SUMO as a binding partner for nuclear proteins. However, the functional consequences of protein SUMOylation remained unclear until two groups independently described that covalent attachment of SUMO to a substrate functioned as a post-translational modification (PTM) (Mahajan et al. 1997; Matunis et al. 1996). During investigations of the nuclear pore complex (NPC), both groups discovered that unmodified Ran-GTPase-activating protein (RanGAP1) was cytosolic and diffuse, but that SUMO-modified RanGAP1 was bound to the NPC through its interaction with a nucleoporin 
protein, RanBP2 (Mahajan et al. 1997). This interaction was also found to protect ran-GAP1 from SUMO-specific protease (SENP)-mediated de-SUMOylation (Zhu et al. 2009). These findings were significant for at least two reasons: (1) this was the first evidence of SUMO as a covalent, reversible protein modifier and (2) SUMO conjugation (SUMOylation) resulted in a change in protein function (i.e., subcellular localization).

Our current knowledge of SUMOylation as a covalent PTM stems from these early studies on nuclear transport. The human genome encodes four SUMO proteins, SUMOs 1-4. SUMO2 and SUMO3 share significantly more sequence similarity to one another than to SUMO1 or SUMO4, and this is reflected in the specificity of SUMOylated targets. For example, SUMO1 and SUMO2/3 appear to have distinct targets (Vertegaal et al. 2006). On the other hand, SUMO4 is predicted to have $87 \%$ amino acid identity with SUMO2 and is expressed in lymph nodes and spleen, although some reports suggest that SUMO4 is a pseudogene (Guo et al. 2004; Hammoudi et al. 2016). The functional capabilities, specificity, and expression profiles of SUMO4 remain unclear. SUMO1 is the most divergent, sharing only $50 \%$ sequence identity with SUMO2/3 (Gareau and Lima 2010). Additional studies on cell type, subcellular localization, protein substrates, and disease versus normal state are required to understand how different SUMOs function.

SUMO proteins are expressed in a precursor form that requires C-terminal proteolytic processing by SUMOspecific proteases (SENP) to expose a diglycine motif essential for conjugation to the target protein (Hickey et al. 2012). There are six mammalian SENP family members (SENP1-3 and SENP5-7), each with distinct substrate specificity and tissue distribution (Kolli et al. 2010). The conjugation of mature SUMO1 and SUMO2/3 to protein substrates is mediated by a pathway similar to ubiquitin and consists of E1, E2, and E3 enzymes (Fig. 1). Mature SUMO is activated by the E1-activating enzyme, a heterodimer composed of SUMO-activating enzymes (SAE) 1 and SAE2 (Desterro et al. 1999; Okuma et al. 1999). SUMO is then transferred from the E1 heterodimer-activating enzyme to the E2-conjugating enzyme, Ubc9 (Lee et al. 1998; Okuma et al. 1999). Finally, it is transferred to substrates with or without catalytic assistance of SUMO E3 ligases (Reverter and Lima 2005). Extensive analysis of several SUMO substrates confirmed that most SUMOaccepting lysine residues $(\mathrm{K})$ lie within a consensus sequence, $\Psi$ KXE, with $\Psi$ representing an aliphatic amino acid, preferably leucine, isoleucine, or valine (Johnson 2004). Other studies reported that this SUMOylation consensus sequence is sufficient to target some substrates, such as RanGAP, through direct interaction with the SUMOconjugating enzyme Ubc9 (Sampson et al. 2001). However, since only one E2-conjugating enzyme exists in the SUMO pathway, SUMO E3 ligases are required to modify other substrates, including SUMOylated at non-consensus sites. On the other hand, the ability of SUMO4 to conjugate to substrates in vivo is still questioned, as SENPs are unable to process the SUMO4 precursor protein (Guo et al. 2004; Owerbach et al. 2005).

SUMO can be attached to substrates as a single SUMO moiety (mono-SUMOylation), at multiple lysine residues (multi-SUMOylation), or consist of a chain of SUMOs attached to one lysine residue (poly-SUMOylation); SUMO2/3 forms polymeric chains, in which SUMO-SUMO linkages occur at $\Omega \mathrm{KXE}$ sequences in their $\mathrm{N}$-terminal extensions (Fig. 1) (Johnson 2004; Tatham et al. 2001). SUMOylation may affect protein localization, stability, and/or activity by altering the dynamics of protein-protein interactions (Johnson 2004). These interactions are primarily mediated by the recruitment of binding partners that harbor SUMO-interacting motifs (SIMs) and are characterized by a stretch of acidic and/or serine residues and a hydrophobic core (Minty et al. 2000; Song et al. 2004). SUMOylation is a highly dynamic and reversible process, and the SENP family of proteins mediates the removal of SUMO from conjugated proteins, the same proteins that are required for the production of mature SUMO (described above). As a result, SENP enzymes maintain the balance between SUMO processing and conjugation/deconjugation. SUMOylation plays significant roles in development, signal transduction, epigenetic processes, and DNA repair (Qu et al. 2014; Sarangi and Zhao 2015; Smith et al. 2011).

Dysregulated SUMOylation has been implicated in several disease states, including cancer, neurodegeneration, and viral infection. In prostate cancer, overexpression of SUMO isopeptidases is accompanied by cellular stress (Bawa-Khalfe et al. 2010; Han et al. 2010; Kaikkonen et al. 2009). In neurodegenerative diseases, including Huntington's, Parkinson's, and Alzheimer's diseases, SUMOylation increases plaque formation and aggregation of key proteins like APP and Tau, contributing to neuronal cytotoxicity (Princz and Tavernarakis 2020; Sarge and Park-Sarge 2009). Several studies report SUMOylation as an essential post-translational mechanism for successful viral infection (Gurer et al. 2005; Lamsoul et al. 2005; Yueh et al. 2006). In addition to viral proteins being SUMO substrates, there is evidence that viruses can manipulate the SUMO pathway to modulate host antiviral responses and promote viral replication and pathogenesis (Lowrey et al. 2017). For example, viruses including Epstein-Barr virus (EBV), hepatitis virus, human immunodeficiency virus (HIV), and herpesviruses (HSV) are among the numerous known viral infections that take advantage of host SUMOylation machinery to promote infection and replication (Brown et al. 2016; Conn et al. 2016; Li et al. 2012; Schaller et al. 2011; Sengupta et al. 2017). Given the complexity of the SUMO pathway, the diverse processes that SUMOylation can affect, and its importance in health and 


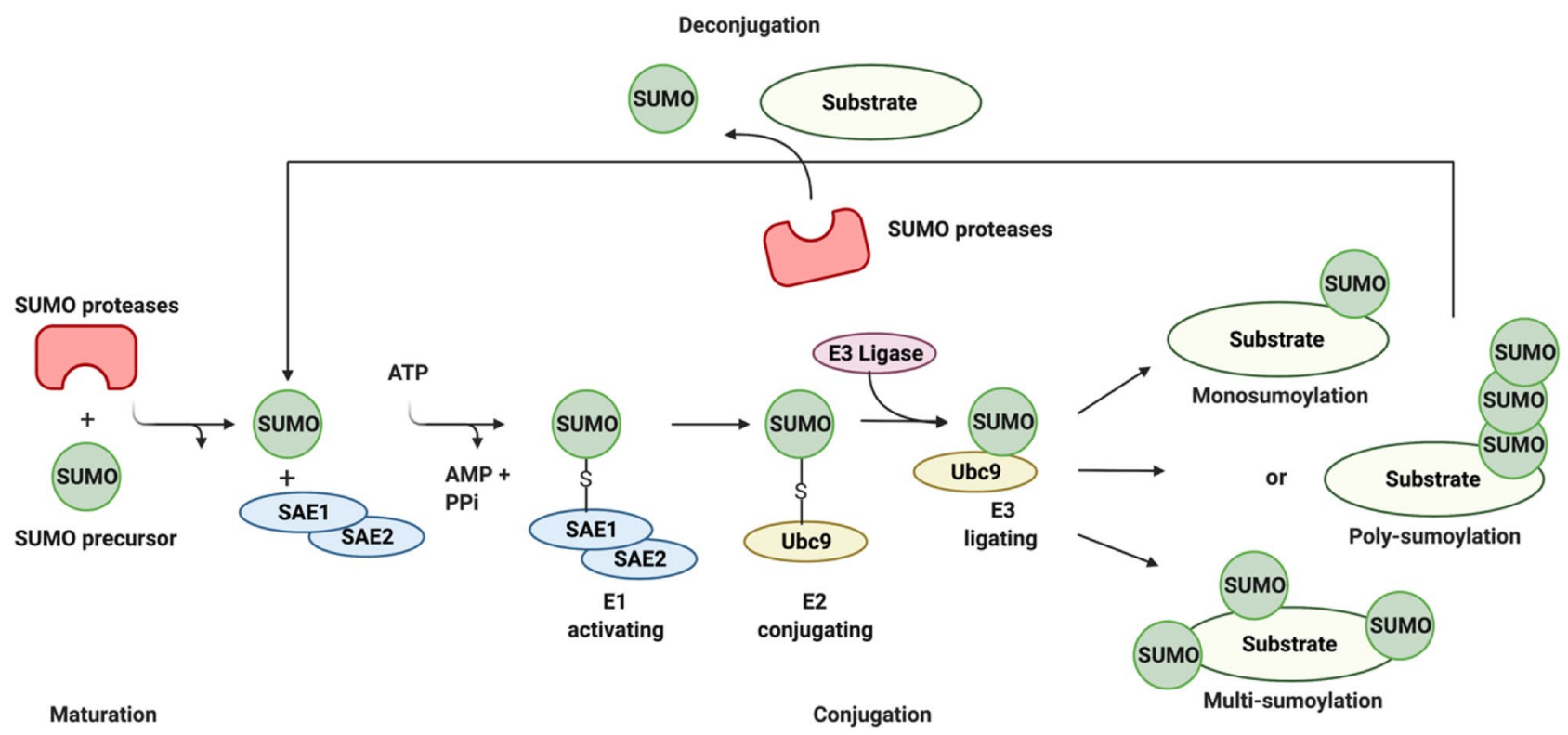

Fig. 1 SUMOylation: the Small Ubiquitin-related Modifier (SUMO) conjugation-deconjugation pathway. SUMO proteins are processed by a SUMO-specific protease to expose a diglycine (-GG) motif, resulting in a mature SUMO peptide $(\sim 12 \mathrm{kDa})$. Mature SUMO is activated in an ATP-dependent reaction, creating an intermediate thioester bond between the active site of SUMO and the heterodimeric E1-activating enzyme (Aos1/Uba2). Following activation, SUMO

disease, increased understanding of the potential impacts of substrate/SUMO status on host response to challenge is critical for developing interventions. Thus, in this review, we describe the implications of SUMO in innate immunity and then focus on the various ways that viral pathogens have evolved to hijack the conserved SUMO machinery. This review concentrates on some of the mechanisms by which viruses manipulate the enzymatic components (E1, E2, E3) of the host SUMOylation machinery to promote viral replication and pathogenesis.

\section{SUMOylation and innate immunity}

The innate immune system is the first line of defense against viral infection. It plays a vital role in early detection, initiating interferon (IFN) synthesis, and promoting an antiviral environment. IFN is primarily secreted by activated T-cells and binds to interferon receptors on infected cells, activating the Jak/STAT pathway and the subsequent expression and activity of interferon-stimulated genes (ISGs) (Robertsen 2018; Tau and Rothman 1999). SUMOylation is suggested to play a role in regulating innate immunity by altering the production of type I interferons (Kim and Ahn 2015; Liu, et al. 2013a, b). Protein inhibitor of activated STAT-1 (PIAS1), a negative regulator of NF- $\mathrm{\kappa B}$ signaling, was also identified as a putative is transferred from the E1 enzyme to Ubc9, the central E2 enzyme. Ubc9 may then directly facilitate the binding of SUMO to the target substrate (not shown), or may recruit an E3 enzyme, which functions in substrate recognition and specificity. SUMO is removed from target proteins (deconjugation) by SUMO proteases, making SUMOylation a reversible process. AMP adenosine monophosphate, PPi pyrophosphate, SAE1/SAE2 SUMO-activating enzyme

SUMO E3 ligase, further implicating SUMOylation as a modulator of innate immunity (Liu et al. 2005). Additional analyses revealed that SUMO-conjugating enzyme Ubc9 conjugated SUMO1 to IкB $\alpha$ (Desterro et al. 1998). Remarkably, SUMOylation of IкB $\alpha$ occurs at lysine 21 residue, which is also required for its ubiquitination. Thus, SUMO1-modified $\mathrm{I} \kappa \mathrm{B} \alpha$ is resistant to ubiquitin-mediated proteasomal degradation (Desterro et al. 1998), providing early evidence for viral manipulation of host SUMO machinery. This antagonistic effect of SUMOylation results in the inhibition of $\mathrm{NF}-\mathrm{\kappa B}$ transcription. Conversely, SUMO2/3 conjugation to NF- $\kappa \mathrm{B}$ essential modifier (NEMO/IKK $\gamma$ ) prevents binding of the deubiquitinase CYLD and potentiates IKK activation, leading to constitutive activation of the NF- $\mathrm{\kappa B}$ pathway (Liu et al. 2013a, b). This modification is reversed explicitly by the de-SUMOylating enzyme SENP6, indicating that the SUMOylation pathway can have both synergistic and antagonistic effects on the ubiquitination of a given substrate. Loss of SENP6 significantly potentiated TLR-mediated NF- $\kappa B$ activation and inflammation (Liu et al. 2013a, b).

Given the dynamic role of protein SUMOylation on innate immunity, it is not surprising that pathogens have evolved to exploit the host SUMOylation machinery. During infection and replication, viruses can use the SUMOylation process to ensure viral persistence and replication. Some viruses mimic SUMO-targeted ubiquitin ligases (STUbLs), 
a group of ubiquitin ligases that contain SUMO-interacting motifs (SIMs), targeting the ligases to SUMOylated proteins (Perry et al. 2008). For example, the HSV early viral protein ICP0 (infected cell polypeptide 0) is responsible for initiating lytic infection. HSV has STUbL properties and targets SUMOylated PML and Sp100 proteins that are important for tumor suppression (Boutell et al. 2003). ICP0 expression in a stable cell line resulted in a decrease in global SUMO conjugates, including SUMOylated forms of PML (Boutell et al. 2011). ICP0 was also shown to target PML more efficiently than SUMO in general (Boutell et al. 2011). These findings suggest that ICP0 counteracts the intrinsic anti-HSV defense by specifically targeting SUMO substrates and inducing proteasomal degradation.

A growing number of viruses have also been shown to target the SUMOylation enzymes to modulate global SUMOylation levels. Some viruses benefit from increased protein SUMOylation, while others benefit from inhibition of SUMOylation processes. For example, the EBV protein kinase BGLF4 suppresses global cellular SUMOylation to enhance extracellular virus production during lytic viral replication ( $\mathrm{Li}$ et al. 2012). In contrast, influenza virus type A (IAV) infection significantly increases the SUMOylation of cellular substrates by both SUMO1 and SUMO2/3 (Domingues et al. 2015). These studies illustrate the importance of viral modulation of global SUMOylation processes during infections. While these studies provide a greater understanding of global changes in SUMOylation during viral infections, it is also essential to understand the specific cellular targets of SUMOylation processes that viruses use to promote proliferation and persistence. Understanding viral manipulation of specific components of the SUMOylation machinery may lead to new therapeutic targets that restore normal SUMOylation levels, thus inhibiting viral replication and pathogenesis.

\section{Viral targeting of SUMOylation enzymes}

\section{Viruses and the E1 SUMO-activating enzyme}

The adenovirus protein Gam 1 is the only example of a viral protein that targets the SUMO-activating SAE1/2 heterodimer (Boggio et al. 2004). Gam1 is critical for viral replication and decreases the histone deacetylase, HDAC1 (Colombo et al. 2002). A later study revealed that Gam1 expression binds to SAE1/2 and inhibits the formation of the E1-SUMO intermediate in vitro (Boggio et al. 2007). Gam1 expression also significantly reduced the intracellular levels of SAE1/2-activating enzymes and the SUMOconjugating enzyme Ubc9, although the mechanism is unknown (Boggio et al. 2007). Gam1 induces the reduction in SAE1/2 by recruiting two cellular ubiquitin ligases to the SAE1/2 complex (Boggio et al. 2007). The ubiquitin ligase ubiquitinates SAE1, resulting in its degradation by the proteasome (Boggio et al. 2007). It was further revealed that the degradation of SAE2 was not directly related to Gam1 but is instead a consequence of SAE1 degradation. The stability of the SUMO E1 heterodimer is closely associated with the presence of both subunits, and the loss of the SAE1 subunit results in proteasome-mediated degradation of SAE2 (Boggio et al. 2007). Consequently, loss of the SUMO E1 heterodimer leads to the accumulation of unmodified SUMO substrates and accumulation of SUMO1 in the cytoplasm, creating a favorable cellular environment for the virus (Colombo et al. 2002). Although the avian adenovirus is currently the only virus reported to target the SUMO-activating enzyme, the capacity to modulate SUMOylation processes by interfering with the E1 heterodimer is likely for multiple viruses.

\section{Viruses and Ubc9}

Given the importance of the only reported E2 SUMOconjugating enzyme in the SUMOylation pathway, Ubc9 is an ideal target for viruses (Fig. 2). Early reports identified the Adenoviral protein E1 A as a binding partner of mouse Ubc9, which potentiates the effects of E1A on PML-NB size and quantity (Hateboer et al. 1996). This finding was significant because it was later discovered that differences in PML-NB size and number correlate with infection states of $\alpha$-herpesviruses (Boutell and Everett 2013). Similarly, interactions with Ubc9 and the HIV-1 envelope protein gp120 stabilizes gp120, resulting in increased viral infectivity of HIV-1 (Jaber et al. 2009). In addition, during latent viral infections of EBV, latent membrane protein 1 (LMP1) interacts with Ubc9 resulting in increased SUMOylation of cellular proteins and subsequent modulation of innate immune responses (Bentz et al. 2011, 2012).

Many viral proteins are SUMOylated and target Ubc9 to facilitate their modification and subsequent localization and/or activity. For example, during human cytomegalovirus (HCMV) infection, the DNA polymerase subunit UL44 binds Ubc 9 and is significantly modified by both SUMO1 and SUMO2/3, resulting in decreased UL44 expression and increased viral replication (Sinigalia et al. 2012). The nucleocapsid protein (NP) of Hantavirus mediates viral assembly of structural proteins, interacts with Ubc9, and is SUMOylated (Alfadhli et al. 2001). Similar to UL44, NP SUMOylation alters its subcellular localization and influences Hantavirus replication (Alfadhli et al. 2001; Maeda et al. 2003). In addition, the human papillomavirus (HPV) protein E2, which supports viral replication, also interacts with Ubc9 (Wu et al. 2008). The E2/ubc9 interaction results in the SUMOylation and increased transcriptional activity of HPV E2 (Wu et al. 2008). On the other hand, 


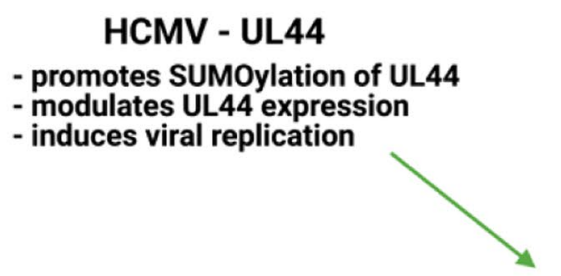

EBV - LMP1

- increases SUMOylation of cellualr proteins

- modulates innnate immune responses
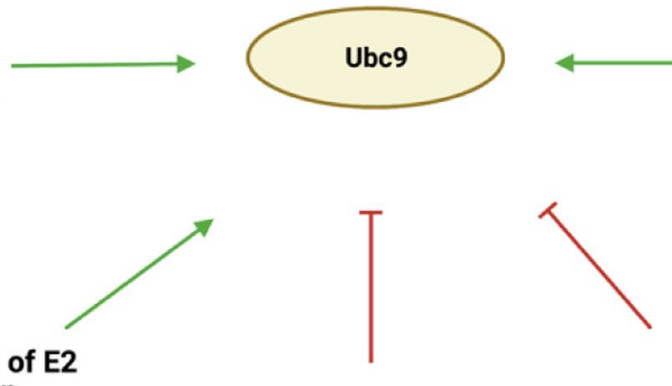

\section{Adenovirus - E1A \\ - inhibits SUMOylation of other cellular substrates - alters the physical properties of PML-NBs}

\section{Adenovirus- Gam1 \\ HPV - E6 \\ - mediates the degradation of Ubc9}

Fig. 2 Viral targeting of Ubc9. ADV Gam1 and HPV E6 induce the degradation of Ubc9, promoting viral replication. ADV E1A interaction with Ubc9 blocks SUMOylation of other cellular substrates and restricts innate immune responses. Conversely, EBV LMP1, HCMV UL44, Hantavirus NP, HPV E2, and HIV gp120 increase

some viruses target Ubc9 to induce the degradation of this crucial SUMO-conjugating enzyme. As previously mentioned, the adenovirus protein Gam 1 expression also greatly reduced Ubc9 stability, promoting transcriptional activation and a favorable cellular environment for viral replication.

In addition, human papillomavirus (HPV) E6 protein targets Ubc9, resulting in proteasome-mediated degradation of Ubc9 (Heaton et al. 2011).

The nucleocapsid $(\mathrm{N})$ protein of severe acute respiratory syndrome coronavirus (SARS-CoV) was also identified as an interacting partner of Ubc9 (Fan et al. 2006; Li et al. 2005). The coronavirus $\mathrm{N}$ protein is a multifunctional protein that plays an essential role in replicating genomic RNA and nucleocapsid assembly (Chang and Brian 1996). Biochemical analysis revealed that SUMO1 modified the $\mathrm{N}$ protein at the lysine 62 residue, resulting in the homooligomerization of the N protein ( $\mathrm{Li}$ et al. 2005). Given that homo-oligomerization is essential for the proper activity of the $\mathrm{N}$ protein, SUMOylation of this protein could play a functional role in SARS-CoV replication (Fan et al. 2006). It is not known whether the N protein of SARS-CoV-2, which causes COVID-19, is also SUMOylated. However, given the significant similarity of the $\mathrm{N}$ protein sequence among coronaviruses, it is likely that the SARS-CoV-2 N the SUMOylation of cellular substrates, modulating viral replication. ADV adenovirus, Gam1; HPV human papilloma virus; EBV EpsteinBarr virus; LMP1 latent membrane protein 1; HCMV human cytomegalovirus; NP nucleocapsid protein

protein is modified by SUMO (Marra et al. 2003; Zhou et al. 2020). Notably, the N protein can stimulate strong immune responses, indicating the $\mathrm{N}$ protein as a potential target for vaccine development.

There is still much to be understood as to how different viral proteins influence ubc9. Although several viral proteins target Ubc9 and are themselves substrates for SUMOylation, how these interactions affect the signaling pathways of the viral protein and Ubc9 requires further investigation. It is possible that in addition to direct interactions with Ubc9, viral proteins can target regulators of Ubc9. Deciphering the many ways viral proteins alter the expression and function of Ubc9 may identify potential targets for the treatment of viral-induced pathologies.

\section{Viruses and SUMO E3 ligases}

Substrate specificity is determined by SUMO E3 ligases and is essential in mediating conjugation to non-consensus lysine residues. However, SUMO E3 ligases are not always required for SUMOylation processes. Still, several viruses can manipulate SUMO E3 ligases to regulate viral replication (Fig. 3). The protein inhibitor of activated STATS (PIAS) family and RANB2 are among the most studied viral targeted SUMO E3 ligases. PIAS family members 


\section{E3 ligases}

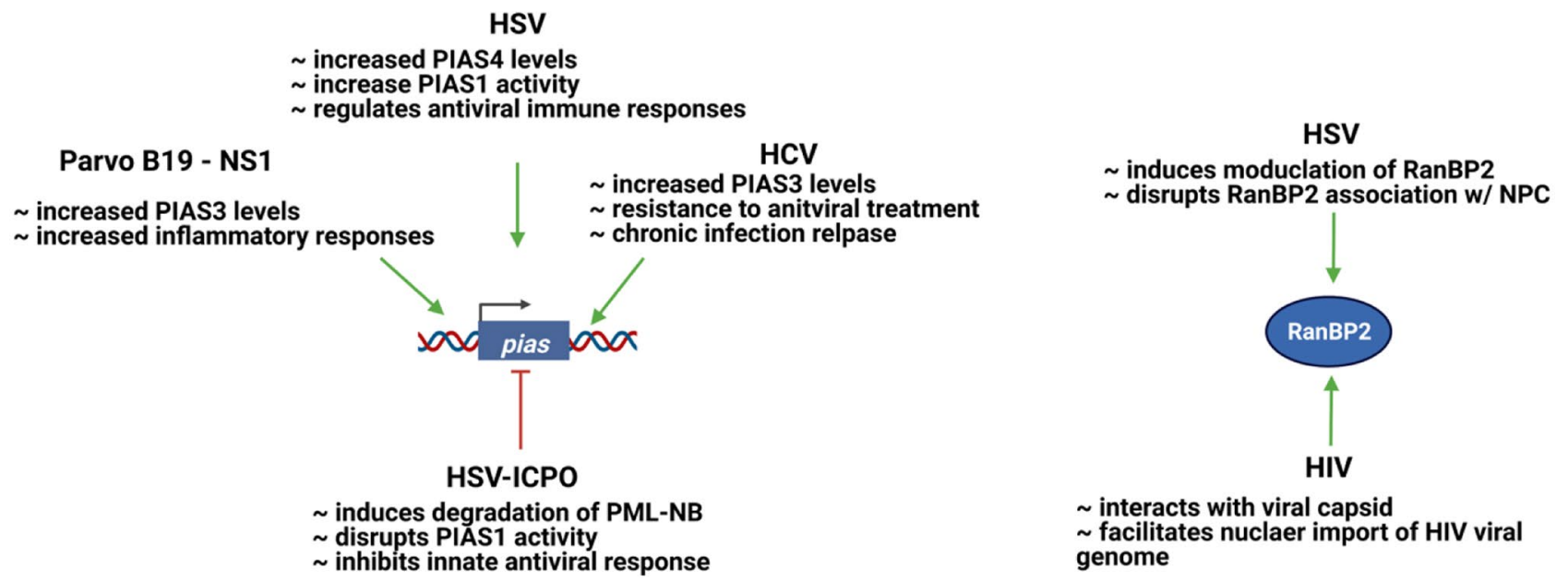

Fig. 3 Viral targeting of SUMO E3 ligases. The HSV ICP0 protein disrupts the activity of PIAS family members and counteracts innate immune responses. Parvovirus B19, HSV, and HCV induce the expression of PIAS1, 3, and/or 4 resulting in regulation of host immune responses and inflammation. The HIV capsid protein interacts with RanBP2 to facilitate nuclear import of HIV DNA. HSV

participate in several cellular processes, including immune regulation, DNA repair, and cell proliferation (Shuai and Liu 2005). During HSV-1 infection, SUMO modifications play a role in PML-NB-mediated intrinsic antiviral immunity. PIAS4 is recruited to nuclear foci containing the viral genome to positively regulate antiviral immune responses to HSV-1 (Conn et al. 2016). Similarly, PIAS1 is recruited to sites adjacent to HSV/PML-NB associated protein colocalization (Brown et al. 2016). This recruitment of constituent antiviral PML-NB factors correlates well with a cooperative restriction of viral gene expression (Everett et al. 2008; Glass and Everett 2013). The innate antiviral response to HSV-1 infection is guided by SUMO-dependent recruitment of PIAS4 and PIAS1. HSV-1 counteracts this component of host immunity via the expression of ICP0, the viral E3 ubiquitin ligase with SUMO-targeted ubiquitin ligase (STUbL)like properties (Boutell et al. 2011). ICP0 induces proteasomal-mediated degradation of PML-NB-associated factors, inhibiting the cellular restriction of viral gene expression (Boutell and Everett 2013).

Conversely, contrasting roles of PIAS as positive regulators of the innate antiviral immune response are reported, as well. Overexpression of duck PIAS2 (duPIAS2) promotes viral replication of the highly pathogenic avian influenza virus (HPAIV) $\mathrm{H}_{5} \mathrm{~N}_{1}$ ( $\mathrm{Zu}$ et al. 2020). PIAS2 influences papillomavirus replication in mammalian cells by interacting with the viral helicase E1 protein, stimulating its SUMOylation (Rosas-Acosta et al. 2005). The parvovirus interacts with RanBP2 and disrupts its association with other NPC members, but the overall functional consequence has not been described. HSV herpes simplex virus, ICP0 infected cell polypeptide 0 , PIAS protein inhibitor of activated STAT, HCV hepatitis $\mathrm{C}$ virus, NS1 nonstructural protein

B19 viral protein NS1 transactivates several cellular promoters, including pias3, and elevated PIAS3 levels are implicated in exacerbated inflammatory responses and endothelial cell dysfunction during infection (Duechting et al. 2008). Increased PIAS3 levels are also associated with relapse of chronic hepatitis $\mathrm{C}$ viral (HCV) infection and resistance to antiviral treatment (Heim et al. 1999). Taken together, these studies revealed a dynamic role of PIAS proteins as intrinsic factors that can restrict or promote viral infection.

While fewer studies address the viral manipulation of the nuclear pore protein RanBP2 compared to PIAS family members, the viral-induced modulation of RanBP2 is thought to regulate the shuttling of proteins and viral genomes into and out of the nucleus. HSV infection induces the modulation of RanBP2 by disrupting its association with other members of the nuclear pore complex (Hofemeister and O'Hare 2008). RanBP2 is also essential for the nuclear import of the HIV-1 viral genome via interactions with the viral capsid (Bichel et al. 2013; Zhang et al. 2010). Consistent with this finding, specific RanBP2 mutations bear strong signatures of virus-driven positive selection during primate evolution (Meyerson et al. 2014). Mutations of a positively selected residue in RanBP2 were shown to affect its interactions with the HIV capsid. However, the mechanistic link between capsid-RanBP2 binding and the nuclear import of the viral genome is unknown (Schaller et al. 2011). 


\section{Viruses and SENPs}

SENPs are the central element of the SUMOylation machinery as they are responsible for regulating the deconjugation of SUMO from its substrates (de-SUMOylation) and overall levels of free SUMO. However, limited data exist on the effects of viral manipulation of SENPS (Fig. 4). In hepatitis B virus (HBV)-infected cells, the HBV X protein (HBx) alters crucial cellular pathways and promotes de-SUMOylation of the host transcription factor Sp110 (Sengupta et al. 2017). Mechanistically, HBx promotes the formation of an Sp110-SENP1-HBx complex, thereby translocating the Sp110 transcription factor to reprogram host gene expression and promote viral proliferation (Sengupta et al. 2017). Kaposi's sarcoma-associated herpesvirus (KHSV) uses a more intricate mechanism that exploits both SUMOylation and de-SUMOylation machinery to promote its replication. During the latent phase, the latent-associated nuclear antigen (LANA) interacts with SUMO2/3 modified proteins to silence viral gene expression (Cai et al. 2013). Interestingly, LANA itself is SUMOylated, and its expression levels are explicitly regulated by SENP6 (Lin et al. 2017). Chromatin immunoprecipitation coupled with sequencing (ChIP-seq) experiments revealed that LANA binds the SENP6 promoter, repressing SENP6 expression (Lin et al. 2017). SENP6 overexpression impeded the establishment of latency as a result of the decrease in the abundance of LANA. These data suggest that LANA inhibits SENP6 from regulating its own SUMOylation and expression levels as a means of maintaining KHSV latency (Lin et al. 2017).

The previously discussed EBV oncoprotein LMP1 also inhibits SUMO-protease activity, resulting in the accumulation of SUMOylated proteins (Selby et al. 2019). Specifically,
LMP1 induces the SUMOylation of the SUMO-protease SENP2 at lysine residues K48 and K447, resulting in reduced SENP2 function, trafficking, and stability (Selby et al. 2019). Interestingly, while mutations of SENP2 K447 reversed LMP1-mediated inhibition of SENP2 activity, mutation of SENP2 K48 failed to abrogate LMP1-mediated inhibition. However, SENP2 localization and stability were not altered when SENP2 K48 and/or K447 were mutated, demonstrating that targeting these residues by LMP1 is not required for SENP2 turnover or distribution (Selby et al. 2019). However, these effects appeared to depend on a functional C-terminal activating region (CTAR) domain of LMP1. Researchers examined if SENP2 was ubiquitinated during EBV latency to better understand this variance, given that SUMO can, directly and indirectly, compete with ubiquitin (Kerscher et al. 2006; Kroetz 2005). Lower levels of ubiquitinated SENP2 were detected in LMP1-expressing lysates as compared to control-expressing lysates. Inhibition of ubiquitination processes also exhibited the observed changes in SENP2 biology as LMP1-mediated SUMOylation (Selby et al. 2019). These results demonstrate that LMP1 modulates the activity, stability, and trafficking of SENP2 by manipulating both SUMOylation and ubiquitin machinery during latent EBV infections.

Much like LANA's role in KHSV latency, these findings highlight the importance of post-translational modifications in latent viral infections. However, the functional role of PTMs like SUMOylation in viral latency remains largely unexplored. For example, the co-repressor COUP-TF interacting protein 2 (CTIP2) recruits a multienzymatic complex to the HIV-1 promoter, preventing the transcription of integrated viral DNA (Marban et al.

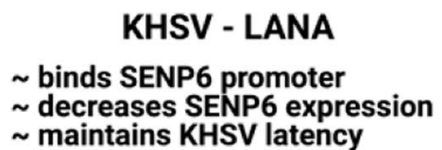

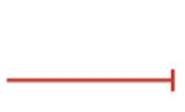
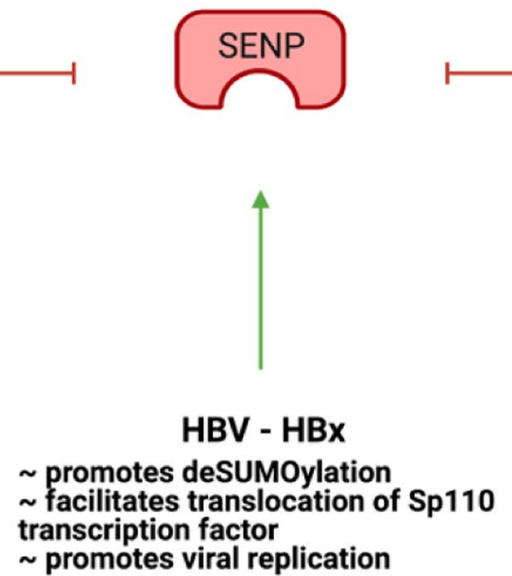

Fig. 4 Viral targeting of SUMO-specific proteases (SENPs). KHSV LANA and EBV LMP1 block cellular SENP by inhibiting its transcription and facilitating its degradation, respectively. The HBV HBx protein induces SENP activity and facilitates de-SUMOylation of cellular substrates. KHSV Kaposi's sarcoma-associated herpesvirus, LANA latency-associated nuclear antigen, EBV Epstein-Barr virus, LMP1 latent membrane protein 1, SENP SUMO-specific protease, $\mathrm{HBV}$ hepatitis $\mathrm{B}$ virus, $\mathrm{HBx}$ hepatitis $\mathrm{B}$ virus protein $\mathrm{X}$ 
2007). However, the mechanisms associated with establishing and maintaining this complex at the HIV-1 promoter remain unclear. Studies have shown that this major regulator protein, CTIP2, is SUMOylated by SUMO1 and that PTMs, including phosphorylation and SUMOylation, mediate its interactions with other proteins and complexes (Dubuissez et al. 2016; Tirard et al. 2012). Specifically, CTIP2 contains a motif, also known as a phosphorylationdependent SUMOylation motif (PDSM), that, when phosphorylated, leads to enhanced SUMOylation of CTIP2 (Hietakangas et al. 2006). The SUMOylation of CTIP2 ultimately results in its proteasomal degradation (Zhang et al. 2012). This "SUMO switch" for CTIP2-mediated transcriptional repression of HIV-1 in microglia or other target cells is of great interest.

Maintaining viral latency in cells with incorporated viral DNA is essential to prevent reactivation. In the case of people with HIV (PWH), lifelong antiretroviral therapy (ART) is required, as latently infected cells of the CNS are from time to time reactivated and can produce virus or viral proteins (Wallet et al. 2019). Previous studies investigating HIV-l latency in microglia found that the tripartite motif-containing 28 (TRIM28) protein, a known SUMO E3 ligase, associates with CTIP2, a known SUMO substrate, and contributes to the establishment and persistence of HIV-1 latency (Ait-Ammar et al. 2021; Ivanov et al. 2007). Mechanistically, CTIP2 directs Tat relocation from the Tat/ TAR complex to the viral latency complex, while TRIM28 interacts and colocalizes with Tat to promote its degradation via the proteasome pathway (Ait-Ammar et al. 2021). Interestingly, a recent study has shown that HIV-1 Vpr mediates the depletion of CTIP2 to counteract viral gene silencing (Forouzanfar et al. 2019). Whether Vpr hijacks the SUMOylation machinery to promote the degradation of CTIP2 is unknown. The recognition of TRIM28 as a SUMO E3 ligase and CTIP2 as a SUMO substrate may have profound therapeutic implications.

\section{Summary}

Since its discovery, SUMO modification has been established as an important regulator of several biological pathways. Here, we have outlined the implications of SUMOylation in innate immunity and the interplay between viruses and the host SUMOylation machinery. Given that SUMOylation helps regulate antiviral immune responses, pathogens have evolved various means of subverting this innate host defense. Viruses have adapted several mechanisms to manipulate different steps of the SUMOylation machinery for their benefit. A growing number of viral proteins have been identified that can control SUMOylation, both on global and substrate-specific levels. Numerous studies have addressed the manipulation of these targets as a regulatory adaptation for viral replication and pathogenesis. However, it is clear that viruses target the SUMOylation machinery to modulate both lytic and lysogenic stages of their life cycle.

In most cases, the overall effect of viral manipulation of host SUMOylation pathways is unclear. Many unidentified viral proteins are likely capable of impacting host protein SUMOylation pathways. In fact, the role of PTMs, like SUMOylation, during latent infections has also been largely unexplored. Identifying these players will provide novel insight into the regulation of viral pathogenesis with the potential to uncover new targets for antiviral therapies.

Abbreviations ADV: Adenovirus; ART: Antiretroviral therapy; CTAR: C-terminal activating region; CTIP2: COUP TF-interacting protein 2; CYLD: CYLD lysine 63 deubiquitinase; gp120: Envelope glycoprotein 120; EBV: Epstein-Barr virus; HBV: Hepatitis B virus; HBx: Hepatitis B virus X protein; HCV: Hepatitis C virus; HSV: Herpes Simplex virus; HPAIV: Highly pathogenic avian influenza virus; HCMV: Human cytomegalovirus; HIV: Human immunodeficiency virus; HPV: Human papillomavirus; ICP0: Infected cell polypeptide 0; IKK: IkB kinases; KHSV: Kaposi's sarcoma-associated herpesvirus; LMP1: Latent membrane protein 1; LANA: Latent-associated nuclear antigen; NEMO: NF-kB essential modulator; NF-kB: Nuclear factor kappa-light-chain-enhancer of activated B cells; IkBa: Nuclear factor of kappa light polypeptide gene enhancer in B-cells inhibitor, alpha; NPC: Nuclear pore complex; NP: Nucleocapsid protein; PDSM: Phosphorylation-dependent SUMOylation motif; PTM: Post-translational modification; PML-NB: Promyelocytic leukemia protein nuclear bodies; PIAS: Protein inhibitor of activated STATS; RanBP2: RAN binding protein 2; RanGAP1: Ran-GTPase activating protein; RING: Really Interesting New Gene; SARS-CoV: Severe acute respiratory syndrome coronavirus; SUMO: Small ubiquitin-related modifier; Sp100: Speckled $110 \mathrm{kDa}$; SAE1/2: SUMO-activating enzyme 1 and 2; SIM: SUMOinteracting motifs; SENP: SUMO-specific protease; TLR: Toll-like receptor; Ubc9: Ubiquitin-conjugating 9

Acknowledgements R01 MH107340-01A1, P01DA037830-01A1, Pennsylvania Department of Health (SAP\# 4100083099) and T32MH079785, the Lemole Integrated Center for Lymphatics Research at LKSOM, Temple University are acknowledged.

\section{Declarations}

Conflicts of interest The authors declare no competing interests.

\section{References}

Ait-Ammar A, Bellefroid M, Daouad F, Martinelli V, Van Assche J, Wallet C, Rodari A, De Rovere M, Fahrenkrog B, Schwartz C, Van Lint C, Gautier V, Rohr O (2021) Inhibition of HIV-1 gene transcription by KAP1 in myeloid lineage. Sci Rep 11(1):2692. https://doi.org/10.1038/s41598-021-82164-w

Alfadhli A, Love Z, Arvidson B, Seeds J, Willey J, Barklis E (2001) Hantavirus nucleocapsid protein oligomerization. J Virol 75(4):2019-2023. https://doi.org/10.1128/JVI.75.4.2019-2023. 2001

Bawa-Khalfe T, Cheng J, Lin SH, Ittmann MM, Yeh ETH (2010) SENP1 induces prostatic intraepithelial neoplasia through multiple mechanisms. J Biol Chem 285(33):25859-25866. https://doi. org/10.1074/jbc.M110.134874 
Bentz GL, Shackelford J, Pagano JS (2012) Epstein-Barr virus latent membrane protein 1 regulates the function of interferon regulatory factor 7 by inducing its sumoylation. J Virol 86(22):12251. https:// doi.org/10.1128/JVI.01407-12

Bentz GL, Whitehurst CB, Pagano JS (2011) Epstein-Barr virus latent membrane protein 1 (LMP1) C-terminal-activating region 3 contributes to LMP1-mediated cellular migration via its interaction with Ubc9. J Virol 85(19):10144-10153

Bichel K, Price AJ, Schaller T, Towers GJ, Freund SMV, James LC (2013) HIV-1 capsid undergoes coupled binding and isomerization by the nuclear pore protein NUP358. Retrovirology 10:81. https://doi.org/10.1186/1742-4690-10-81

Boddy M, Howe K, Etkin L, Solomon E, Freemont P (1996) PIC 1 a novel ubiquitin-like protein which interacts with the PML component of a multiprotein complex that is disrupted in acute promyelocytic leukaemia. Oncogene 13:971-982

Boggio R, Colombo R, Hay RT, Draetta GF, Chiocca S (2004) A mechanism for inhibiting the SUMO pathway. Mol Cell 16(4):549-561. https://doi.org/10.1016/j.molcel.2004.11.007

Boggio R, Passafaro A, Chiocca S (2007) Targeting SUMO E1 to ubiquitin ligases: a viral strategy to counteract sumoylation*. J Biol Chem 282(21):15376-15382. https://doi.org/10.1074/jbc. M700889200

Boutell C, Cuchet-Lourenço D, Vanni E, Orr A, Glass M, McFarlane S, Everett RD (2011) A viral ubiquitin ligase has substrate preferential SUMO targeted ubiquitin ligase activity that counteracts intrinsic antiviral defence. PLoS Pathog 7(9). https://doi.org/10. 1371/journal.ppat.1002245

Boutell C, Everett RD (2013) Regulation of alphaherpesvirus infections by the ICP0 family of proteins. J Gen Virol 94(3):465481. https://doi.org/10.1099/vir.0.048900-0

Boutell C, Orr A, Everett RD (2003) PML residue lysine 160 is required for the degradation of PML induced by Herpes Simplex virus type 1 regulatory protein ICP0. J Virol 77(16):8686-8694. https://doi.org/10.1128/JVI.77.16.8686-8694.2003

Brown JR, Conn KL, Wasson P, Charman M, Tong L, Grant K, McFarlane S, Boutell C (2016) SUMO ligase Protein Inhibitor of Activated STAT1 (PIAS1) is a constituent promyelocytic leukemia nuclear body protein that contributes to the intrinsic antiviral immune response to Herpes Simplex virus 1. J Virol 90(13):5939-5952. https://doi.org/10.1128/JVI.00426-16

Cai Q, Cai S, Zhu C, Verma SC, Choi JY, Robertson ES (2013) A unique SUMO-2-interacting motif within LANA is essential for KSHV latency. PLoS Pathog 9(11):e1003750. https://doi. org/10.1371/journal.ppat.1003750

Chang RY, Brian DA (1996) Cis requirement for N-specific protein sequence in bovine coronavirus defective interfering RNA replication. J Virol 70(4):2201-2207

Colombo R, Boggio R, Seiser C, Draetta GF, Chiocca S (2002) The adenovirus protein Gam1 interferes with sumoylation of histone deacetylase 1. EMBO Rep 3(11):1062-1068. https://doi.org/10. 1093/embo-reports/kvf213

Conn KL, Wasson P, McFarlane S, Tong L, Brown JR, Grant KG, Domingues P, Boutell C (2016) Novel role for Protein Inhibitor of Activated STAT 4 (PIAS4) in the restriction of Herpes Simplex virus 1 by the cellular intrinsic antiviral immune response. J Virol 90(9):4807-4826. https://doi.org/10.1128/JVI.03055-15

Desterro JMP, Rodriguez MS, Hay RT (1998) SUMO-1 modifica-

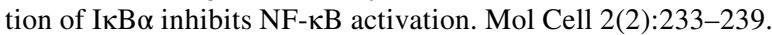
https://doi.org/10.1016/S1097-2765(00)80133-1

Desterro JMP, Rodriguez MS, Kemp GD, Hay RT (1999) Identification of the enzyme required for activation of the small ubiquitin-like protein SUMO-1. J Biol Chem 274(15):10618-10624. https://doi.org/10.1074/jbc.274.15.10618

Domingues P, Golebiowski F, Tatham MH, Lopes AM, Taggart A, Hay RT, Hale BG (2015) Global reprogramming of host
SUMOylation during influenza virus infection. Cell Rep 13(7):1467-1480. https://doi.org/10.1016/j.celrep.2015.10.001

Dubuissez M, Loison I, Paget S, Vorng H, Ait-Yahia S, Rohr O, Tsicopoulos A, Leprince D (2016) Protein kinase C-mediated phosphorylation of BCL11B at serine 2 negatively regulates its interaction with NuRD complexes during CD4+ T-cell activation. Mol Cell Biol 36(13):1881-1898. https://doi.org/10.1128/MCB.00062-16

Duechting A, Tschöpe C, Kaiser H, Lamkemeyer T, Tanaka N, Aberle S, Lang F, Torresi J, Kandolf R, Bock CT (2008) Human parvovirus B19 NS1 protein modulates inflammatory signaling by activation of STAT3/PIAS3 in human endothelial cells. J Virol 82(16):7942-7952. https://doi.org/10.1128/JVI.00891-08

Everett RD, Parada C, Gripon P, Sirma H, Orr A (2008) Replication of ICP0-null mutant Herpes Simplex virus type 1 is restricted by both PML and Sp100. J Virol 82(6):2661-2672. https://doi.org/ 10.1128/JVI.02308-07

Fan Z, Zhuo Y, Tan X, Zhou Z, Yuan J, Qiang B, Yan J, Peng X, Gao GF (2006) SARS-CoV nucleocapsid protein binds to hUbc9 a ubiquitin conjugating enzyme of the sumoylation system. J Med Virol 78(11):1365-1373. https://doi.org/10.1002/jmv.20707

Forouzanfar F, Ali S, Wallet C, De Rovere M,Ducloy C, El Mekdad H, El Maassarani M, Aït-Ammar A, Van Assche J, Boutant E,Daouad F, Margottin-Goguet F, Moog C, Van Lint C, Schwartz C, Rohr O (2019)HIV-1 Vpr mediates the depletion of the cellular repressor CTIP2 to counteractviral gene silencing. Sci Rep 9. https://doi. org/10.1038/s41598-019-48689-x

Gareau JR, Lima CD (2010) The SUMO pathway: emerging mechanisms that shape specificity conjugation and recognition. Nat Rev Mol Cell Biol 11(12):861-871. https://doi.org/10.1038/nrm3011

Glass M, Everett RD (2013) Components of promyelocytic leukemia nuclear bodies (ND10) act cooperatively to repress herpesvirus infection. J Virol 87(4):2174-2185. https://doi.org/10.1128/JVI. 02950-12

Guo D, Li M, Zhang Y, Yang P, Eckenrode S, Hopkins D, Zheng W, Purohit S, Podolsky RH, Muir A, Wang J, Dong Z, Brusko T, Atkinson M, Pozzilli P, Zeidler A, Raffel LJ, Jacob CO, Park Y, Wang CY (2004) A functional variant of SUMO4 a new IкB $\alpha$ modifier is associated with type 1 diabetes. Nat Genet 36(8):837841. https://doi.org/10.1038/ng1391

Gurer C, Berthoux L, Luban J (2005) Covalent modification of human immunodeficiency virus type 1 p6 by SUMO-1. J Virol 79(2):910-917. https://doi.org/10.1128/JVI.79.2.910-917.2005

Hammoudi V, Vlachakis G, Schranz ME, van den Burg HA (2016) Whole-genome duplications followed by tandem duplications drive diversification of the protein modifier SUMO in angiosperms. New Phytol 211(1):172-185. https://doi.org/10.1111/ nph.13911

Han Y, Huang C, Sun X, Xiang B, Wang M, Yeh ETH, Chen Y, Li H, Shi G, Cang H, Sun Y, Wang J, Wang W, Gao F, Yi J (2010) SENP3mediated de-conjugation of SUMO2/3 from promyelocytic leukemia is correlated with accelerated cell proliferation under mild oxidative stress. J Biol Chem 285(17):12906-12915. https://doi.org/10.1074/ jbc.M109.071431

Hateboer G, Hijmans EM, Nooij JBD, Schlenker S, Jentsch S, Bernards $\mathrm{R}$ (1996) MUBC9 a novel adenovirus E1A-interacting protein that complements a yeast cell cycle defect*. J Biol Chem 271(42):2590625911. https://doi.org/10.1074/jbc.271.42.25906

Heaton PR, Deyrieux AF, Bian XL, Wilson VG (2011) HPV E6 proteins target Ubc9 the SUMO conjugating enzyme. Virus Res 158(1):199-208. https://doi.org/10.1016/j.virusres.2011.04.001

Heim MH, Moradpour D, Blum HE (1999) Expression of hepatitis C virus proteins inhibits signal transduction through the Jak-STAT pathway. J Virol 73(10):8469-8475

Hickey CM, Wilson NR, Hochstrasser M (2012) Function and regulation of SUMO proteases. Nat Rev Mol Cell Biol 13(12):755-766. https://doi.org/10.1038/nrm3478 
Hietakangas V, Anckar J, Blomster HA, Fujimoto M, Palvimo JJ, Nakai A, Sistonen L (2006) PDSM a motif for phosphorylation-dependent SUMO modification. Proc Natl Acad Sci 103(1):45-50

Hofemeister H, O'Hare P (2008) Nuclear pore composition and gating in herpes simplex virus-infected cells. J Virol 82(17):8392-8399. https://doi.org/10.1128/JVI.00951-08

Ivanov AV, Peng H, Yurchenko V, Yap KL, Negorev DG, Schultz DC, Psulkowski E, Fredericks WJ, White DE, Maul GG (2007) PHD domain-mediated E3 ligase activity directs intramolecular sumoylation of an adjacent bromodomain required for gene silencing. Mol Cell 28(5):823-837

Jaber T, Bohl CR, Lewis GL, Wood C, West JT, Weldon RA (2009) Human Ubc9 contributes to production of fully infectious human immunodeficiency virus type 1 virions. J Virol 83(20):1044810459. https://doi.org/10.1128/JVI.00237-09

Johnson ES (2004) Protein modification by SUMO. Annu Rev Biochem 73(1):355-382. https://doi.org/10.1146/annurev.biochem. 73.011303 .074118

Kaikkonen S, Jääskeläinen T, Karvonen U, Rytinki MM, Makkonen H, Gioeli D, Paschal BM, Palvimo JJ (2009) SUMO-Specific Protease 1 (SENP1) Reverses the hormone-augmented SUMOylation of androgen receptor and modulates gene responses in prostate cancer cells. Mol Endocrinol 23(3):292-307. https://doi.org/10. 1210/me.2008-0219

Kerscher O, Felberbaum R, Hochstrasser M (2006) Modification of proteins by ubiquitin and ubiquitin-like proteins. Annu Rev Cell Dev Biol 22:159-180. https://doi.org/10.1146/annurev.cellbio.22. 010605.093503

Kim YE, Ahn JH (2015) Positive role of promyelocytic leukemia protein in type i interferon response and its regulation by human cytomegalovirus. PLOS Pathog 11(3):e1004785. https://doi.org/ 10.1371/journal.ppat.1004785

Kolli N, Mikolajczyk J, Drag M, Mukhopadhyay D, Moffatt N, Dasso M, Salvesen G, Wilkinson KD (2010) Distribution and paralog specificity of mammalian deSUMOylating enzymes. Biochem J 430(2):335-344. https://doi.org/10.1042/BJ20100504

Kroetz MB (2005) SUMO: a ubiquitin-like protein modifier. Yale J Biol Med 78(4):197-201

Lamsoul I, Lodewick J, Lebrun S, Brasseur R, Burny A, Gaynor RB, Bex F (2005) Exclusive ubiquitination and sumoylation on overlapping lysine residues mediate NF-kappaB activation by the human T-cell leukemia virus tax oncoprotein. Mol Cell Biol 25(23):10391-10406. https://doi.org/10.1128/MCB.25.23.1039110406.2005

Lee GW, Melchior F, Matunis MJ, Mahajan R, Tian Q, Anderson P (1998) Modification of Ran GTPpase-activating protein by the small ubiquitin- related modifier SUMO-1 requires Ubc9 an E2-type ubiquitin-conjugating enzyme homologue. J Biol Chem 273(11):6503-6507. https://doi.org/10.1074/jbc.273.11.6503

Li FQ, Xiao H, Tam JP, Liu DX (2005) Sumoylation of the nucleocapsid protein of severe acute respiratory syndrome coronavirus. FEBS Lett 579(11):2387-2396. https://doi.org/10.1016/j.febslet. 2005.03.039

Li R, Wang L, Liao G, Guzzo CM, Matunis MJ, Zhu H, Hayward SD (2012) SUMO binding by the Epstein-Barr virus protein kinase BGLF4 is crucial for BGLF4 function. J Virol 86(10):5412-5421. https://doi.org/10.1128/JVI.00314-12

Lin X, Sun R, Zhang F, Gao Y, Bin L, Lan K (2017). The latencyassociated nuclear antigen of Kaposi's sarcoma-associated herpesvirus inhibits expression of SUMO/sentrin-specific peptidase 6 to facilitate establishment of latency. J Virol 91(17). https://doi. org/10.1128/JVI.00806-17

Liu B, Yang R, Wong KA, Getman C, Stein N, Teitell MA, Cheng G, Wu H, Shuai K (2005) Negative regulation of NF- $\kappa B$ signaling by PIAS1. Mol Cell Biol 25(3):1113-1123. https://doi.org/10.1128/ MCB.25.3.1113-1123.2005
Liu X, Chen W, Wang Q, Li L, Wang C (2013a) Negative regulation of TLR inflammatory signaling by the SUMO-deconjugating enzyme SENP6. PLOS Pathog 9(6):e1003480. https://doi.org/10.1371/ journal.ppat. 1003480

Liu X, Wang Q, Chen W, Wang C (2013b) Dynamic regulation of innate immunity by ubiquitin and ubiquitin-like proteins. Cytokine Growth Factor Rev 24(6):559-570. https://doi.org/10.1016/j.cytogfr.2013. 07.002

Lowrey AJ, Cramblet W, Bentz GL (2017) Viralmanipulation of the cellular sumoylation machinery. Cell Commun Signaling 15. https://doi.org/ 10.1186/s12964-017-0183-0

Maeda A, Lee BH, Yoshimatsu K, Saijo M, Kurane I, Arikawa J, Morikawa $S$ (2003) The intracellular association of the nucleocapsid protein (NP) of Hantaan virus (HTNV) with small ubiquitin-like modifier-1 (SUMO-1) conjugating enzyme 9 (Ubc9). Virol 305(2):288-297. https://doi.org/10.1006/viro.2002.1767

Mahajan R, Delphin C, Guan T, Gerace L, Melchior F (1997) A small ubiquitin-related polypeptide involved in targeting RanGAP1 to nuclear pore complex protein RanBP2. Cell 88(1):97-107. https:// doi.org/10.1016/S0092-8674(00)81862-0

Marban C, Suzanne S, Dequiedt F, de Walque S, Redel L, Van Lint C, Aunis D, Rohr O (2007) Recruitment of chromatin-modifying enzymes by CTIP2 promotes HIV-1 transcriptional silencing. EMBO J 26(2):412-423. https://doi.org/10.1038/sj.emboj.7601516

Marra MA, Jones SJ, Astell CR, Holt RA, Brooks-Wilson A, Butterfield YS, Khattra J, Asano JK, Barber SA, Chan SY (2003) The genome sequence of the SARS-associated coronavirus. Sci 300(5624):1399-1404

Matunis MJ, Coutavas E, Blobel G (1996) A novel ubiquitin-like modification modulates the partitioning of the Ran-GTPase-activating protein RanGAP1 between the cytosol and the nuclear pore complex. J Cell Biol 135(6):1457-1470. https://doi.org/10.1083/jcb. 135.6.1457

Meluh PB, Koshland D (1995) Evidence that the MIF2 gene of Saccharomyces cerevisiae encodes a centromere protein with homology to the mammalian centromere protein CENP-C. Mol Biol Cell 6(7):793-807. https://doi.org/10.1091/mbc.6.7.793

Meyerson NR, Rowley PA, Swan CH, Le DT, Wilkerson GK, Sawyer SL (2014) Positive selection of primate genes that promote HIV-1 replication. Virol 454:291-298. https://doi.org/10.1016/j.virol.2014.02.029

Minty A, Dumont X, Kaghad M, Caput D (2000) Covalent modification of p73alpha by SUMO-1: Two-hybrid screening with p73 identifies novel SUMO-1-interacting proteins and a SUMO-1 interaction motif. J Biol Chem 275(46):36316-36323. https:// doi.org/10.1074/jbc.M004293200

Okuma T, Honda R, Ichikawa G, Tsumagari N, Yasuda H (1999) In vitro SUMO-1 modification requires two enzymatic steps E1 and E2. Biochem Biophys Res Commun 254(3):693-698. https:// doi.org/10.1006/bbrc.1998.9995

Okura T, Gong L, Kamitani T, Wada T, Okura I, Wei CF, Chang HM, Yeh ET (1996) Protection against Fas/APO-1- and tumor necrosis factor-mediated cell death by a novel protein sentrin. J Immunol 157(10):4277-4281

Owerbach D, McKay EM, Yeh ETH, Gabbay KH, Bohren KM (2005) A proline-90 residue unique to SUMO-4 prevents maturation and sumoylation. Biochem Biophys Res Commun 337(2):517-520. https://doi.org/10.1016/j.bbrc.2005.09.090

Perry JJP, Tainer JA, Boddy MN (2008) A SIM-ultaneous role for SUMO and ubiquitin. Trends Biochem Sci 33(5):201-208. https:// doi.org/10.1016/j.tibs.2008.02.001

Princz A, Tavernarakis N (2020) SUMOylation in neurodegenerative diseases. Gerontology 66(2):122-130. https://doi.org/10.1159/ 000502142

Qu Y, Chen Q, Lai X, Zhu C, Chen C, Zhao X, Deng R, Xu M, Yuan H, Wang Y (2014) SUMOylation of Grb2 enhances the ERK activity by increasing its binding with Sos1. Mol Cancer 13(1):1-12 
Reverter D, Lima CD (2005) Insights into E3 ligase activity revealed by a SUMO-RanGAP1-Ubc9-Nup358 complex. Nature 435(7042):687-692. https://doi.org/10.1038/nature03588

Robertsen B (2018) The role of type I interferons in innate and adaptive immunity against viruses in Atlantic salmon. Dev Comp Immunol 80:41-52. https://doi.org/10.1016/j.dci.2017.02.005

Rosas-Acosta G, Langereis MA, Deyrieux A, Wilson VG (2005) Proteins of the PIAS family enhance the sumoylation of the papillomavirus E1 protein. Virol 331(1):190-203. https://doi.org/10. 1016/j.virol.2004.10.025

Sampson DA, Wang M, Matunis MJ (2001) The Small Ubiquitinlike Modifier-1 (SUMO-1) consensus sequence mediates Ubc9 binding and is essential for SUMO-1 modification. J Biol Chem 276(24):21664-21669. https://doi.org/10.1074/jbc.M100006200

Sarangi P, Zhao X (2015) SUMO-mediated regulation of DNA damage repair and responses. Trends Biochem Sci 40(4):233-242. https:// doi.org/10.1016/j.tibs.2015.02.006

Sarge KD, Park-Sarge OK (2009) Sumoylation and human disease pathogenesis. Trends Biochem Sci 34(4):200-205. https://doi. org/10.1016/j.tibs.2009.01.004

Schaller T, Ocwieja KE, Rasaiyaah J, Price AJ, Brady TL, Roth SL, Hué S, Fletcher AJ, Lee K, KewalRamani VN, Noursadeghi M, Jenner RG, James LC, Bushman FD, Towers GJ (2011) HIV-1 capsid-cyclophilin interactions determine nuclear import pathway integration targeting and replication efficiency. PLoS Pathog 7(12):e1002439. https://doi.org/10.1371/journal.ppat.1002439

Selby TL, Biel N, Varn M, Patel S, Patel A, Hilding L, Ray A, Ross T, Cramblet WT, Moss CR, Lowrey AJ, Bentz GL (2019) The Epstein-Barr virus oncoprotein LMP1 regulates the function of SENP2 a SUMO-protease. Sci Rep 9(1):9523. https://doi.org/10. 1038/s41598-019-45825-5

Sengupta I, Das D, Singh SP, Chakravarty R, Das C (2017) Host transcription factor Speckled $110 \mathrm{kDa}(\mathrm{Sp} 110)$ a nuclear body protein is hijacked by hepatitis B virus protein $\mathrm{X}$ for viral persistence. J Biol Chem 292(50):20379-20393. https://doi.org/10.1074/jbc. M117.796839

Shen Z, Pardington-Purtymun PE, Comeaux JC, Moyzis RK, Chen DJ (1996) UBL1 a Human ubiquitin-like protein associating with human RAD51/RAD52 proteins. Genomics 36(2):271-279. https://doi.org/10.1006/geno.1996.0462

Shuai K, Liu B (2005) Regulation of gene-activation pathways by PIAS proteins in the immune system. Nat Rev Immunol 5(8):593-605. https://doi.org/10.1038/nri1667

Sinigalia E, Alvisi G, Segré CV, Mercorelli B, Muratore G, Winkler M, Hsiao HH, Urlaub H, Ripalti A, Chiocca S, Palù G, Loregian A (2012) The human cytomegalovirus DNA polymerase processivity factor UL44 is modified by SUMO in a DNA-dependent manner. PLOS ONE 7(11):e49630. https://doi.org/10.1371/journal.pone. 0049630

Smith M, Mallin DR, Simon JA, Courey AJ (2011) Small ubiquitin-like modifier (SUMO) conjugation impedes transcriptional silencing by the polycomb group repressor Sex Comb on Midleg. J Biol Chem 286(13):11391-11400

Song J, Durrin LK, Wilkinson TA, Krontiris TG, Chen Y (2004) Identification of a SUMO-binding motif that recognizes SUMOmodified proteins. Proc Natl Acad Sci U S A 101(40):1437314378. https://doi.org/10.1073/pnas.0403498101
Tatham MH, Jaffray E, Vaughan OA, Desterro JM, Botting CH, Naismith JH, Hay RT (2001) Polymeric chains of SUMO-2 and SUMO-3 are conjugated to protein substrates by SAE1/SAE2 and Ubc9. J Biol Chem 276(38):35368-35374. https://doi.org/ 10.1074/jbc.M104214200

Tau G, Rothman P (1999) Biologic functions of the IFN- $\gamma$ receptors. Allergy 54(12):1233-1251. https://doi.org/10.1034/j.1398-9995. 1999.00099.x

Tirard M, Hsiao HH, Nikolov M, Urlaub H, Melchior F, Brose N (2012) In vivo localization and identification of SUMOylated proteins in the brain of His6-HA-SUMO1 knock-in mice. Proc Natl Acad Sci 109(51):21122-21127. https://doi.org/10.1073/ pnas. 1215366110

Vertegaal ACO, Andersen JS, Ogg SC, Hay RT, Mann M, Lamond AI (2006) Distinct and overlapping sets of SUMO-1 and SUMO-2 target proteins revealed by quantitative proteomics. Mol Cell Proteom 5(12):2298-2310. https://doi.org/10.1074/mcp.M600212-MCP200

Wallet C, De Rovere M, Van Assche J, Daouad F, De Wit S, Gautier V, Mallon PWG, Marcello A, Van Lint C, Rohr O, Schwartz C (2019) Microglial cells: the main HIV-1 reservoir in the brain. Front Cell Infect Microbiol 9:362. https://doi.org/10.3389/fcimb.2019.00362

Wu YC, Roark AA, Bian XL, Wilson VG (2008) Modification of papillomavirus E2 proteins by the small ubiquitin-like modifier family members (SUMOs). Virol 378(2):329-338. https://doi.org/10. 1016/j.virol.2008.06.008

Yueh A, Leung J, Bhattacharyya S, Perrone LA, de los Santos K, Pu SY, Goff SP, (2006) Interaction of moloney murine leukemia virus capsid with Ubc9 and PIASy mediates SUMO-1 addition required early in infection. J Virol 80(1):342-352. https://doi.org/10.1128/ JVI.80.1.342-352.2006

Zhang L, Bhattacharya S, Leid M, Ganguli-Indra G, Indra AK (2012) Ctip2 is a dynamic regulator of epidermal proliferation and differentiation by integrating EGFR and Notch signaling. J Cell Sci 125(23):5733-5744. https://doi.org/10.1242/jcs.108969

Zhang R, Mehla R, Chauhan A (2010) Perturbation of host nuclear membrane component RanBP2 impairs the nuclear import of human immunodeficiency virus-1 preintegration complex (DNA). PloS One 5(12):e15620. https://doi.org/10.1371/journal.pone.0015620

Zhong S, Müller S, Ronchetti S, Freemont PS, Dejean A, Pandolfi PP (2000) Role of SUMO-1-modified PML in nuclear body formation. Blood 95(9):2748-2752. https://doi.org/10.1182/blood. V95.9.2748.009k31a_2748_2752

Zhou P, Yang XL, Wang XG, Hu B, Zhang L, Zhang W, Si HR, Zhu Y, Li B, Huang CL (2020) A pneumonia outbreak associated with a new coronavirus of probable bat origin. Nat 579(7798):270-273

Zhu S, Goeres J, Sixt KM, Békés M, Zhang XD, Salvesen GS, Matunis MJ (2009) Protection from isopeptidase-mediated deconjugation regulates paralog-selective sumoylation of RanGAP1. Mol Cell 33(5):570-580. https://doi.org/10.1016/j.molcel.2009.02.008

Zu S, Xue Q, He Z, Shi C, Zhang J, Wu W, Li W, Liu Z, Huang J, Jiao P, Liao M (2020) Duck PIAS2 promotes H5N1 avian influenza virus replication through its SUMO E3 ligase activity. Front Microbiol 11:1246. https://doi.org/10.3389/fmicb.2020.01246

Publisher's Note Springer Nature remains neutral with regard to jurisdictional claims in published maps and institutional affiliations. 